\title{
Segmentation of Scanned Insect Footprints Using ART2 for Threshold Selection
}

\author{
Bok-Suk Shin ${ }^{1}$, Eui-Young Cha ${ }^{1}$, Young Woon $\mathrm{Woo}^{2}$, and Reinhard Klette ${ }^{3}$ \\ ${ }^{1}$ Dept. of Computer Science, Pusan National University, Busan, Korea \\ shinbs@pusan.ac.kr, eycha@pusan.ac.kr \\ ${ }^{2}$ Dept. of Multimedia Engineering, Dong-Eui University, Busan, Korea \\ ywwoo@deu.ac.kr \\ ${ }^{3}$ Dept. of Computer Science, The University of Auckland, Auckland, New Zealand \\ r.klette@auckland.ac.nz
}

\begin{abstract}
In a process of insect footprint recognition, footprint segments need to be extracted from scanned insect footprints in order to find out appropriate features for classification. In this paper, we use a clustering method in a preprocessing stage for extraction of insect footprint segments. In general, sizes and strides of footprints may be different according to type and size of an insect for recognition. Therefore we propose a method for insect footprint segment extraction using an improved ART2 algorithm regardless of size and stride of footprint pattern. In the improved ART2 algorithm, an initial threshold value for clustering is determined automatically using the contour shape of the graph created by accumulating distances between all the spots within a binarized footprint pattern image. In the experimental results, applying the proposed method to two kinds of insect footprint patterns, we illustrate that clustering is accomplished correctly.
\end{abstract}

Keywords: Insect footprint segmentation, Clustering, ART2 algorithm.

\section{Introduction}

Modern transportation also means that various kinds of insects change places by vehicle, aircraft or ship. There are no problems in cases where native insects travel within their habitat, but it may cause harm to the ecosystem or the environment if insects enter an area outside of their habitat. In order to monitor movements or presence of insects (e.g., in containers in airplanes or ships, or in defined areas such as an island), special methods have been designed for the monitoring of insects, taking their characteristics into account.

Examples of monitoring devices are illustrated in Figure 1. Such tunnels are widely used for collecting footprints of small animals (such as rats or mice) and of various kinds of insects. The acquired footprints are visually inspected or scanned for automated reading; they are used for various monitoring tasks, for example for verifying the presence of some insects, or for more detailed ecological or biological studies as supported by those footprints [12]. 


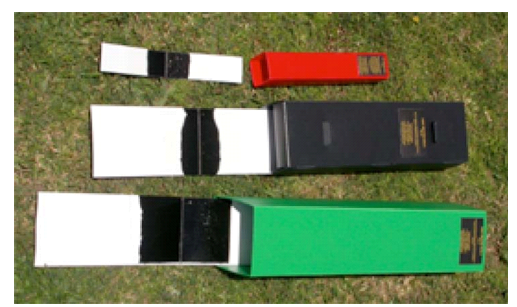

Fig. 1. Tracking tunnel devices of varying sizes using Black Trakka ${ }^{\mathrm{TM}}$ tracking cards 3]: insects or small animals are attracted by a lure to walk into the tunnel, across the inked area, leaving tracks on the white card

The acquired insect footprints, using such tracking tunnel devices for collection, are then typically identified by entomologists having expert knowledge about insect's morphology [4. The identification requires that individual footprints are extracted (e.g., by using morphological features of each kind of insect [5) and then clustered into meaningful track patterns, but it may be hard to extract, analyze and classify insect footprints even for the experienced human specialist if available knowledge about entomology and visible patterns do not match (e.g., if too many insects left traces on the same card).

For automated reading of such cards, we start with a method to extract automatically segments for later classification, with the aim to remove unnecessary human preprocessing, improve time efficiency, and increase accuracy for insect footprint recognition, possibly even for situations where expert knowledge about entomology is not accessible.

In this paper, we propose a method for insect footprint segmentation using an improved ART2 (Adaptive Resonance Theory) algorithm, regardless of size and stride of each type of insect footprint. In the improved ART2 algorithm, the threshold value for clustering is determined automatically using contour shape of the graph created by accumulating distances between all of the "spots" of an insect footprint pattern image scanned from one of those tracking cards. The paper improves a method that was proposed in [6.

\section{Improved Footprint Segmentation}

First, we define four terms for describing our methodology. We define a "spot" as a set of connected pixels in a binarized footprint image and a "region" as a set of spots for each foot. And then we define a "segment" as a set of three regions for front foot, mid foot and hind foot and a "pattern" as a set of segments in a footprint image. This paper considers the following steps for collecting tracks, scanning, and extracting footprint segments (For a graphical sketch of the overall process, see Figure 2.):

Step 1: Insect footprints are acquired on tracking cards, placed into tracking tunnel devices for some time. 


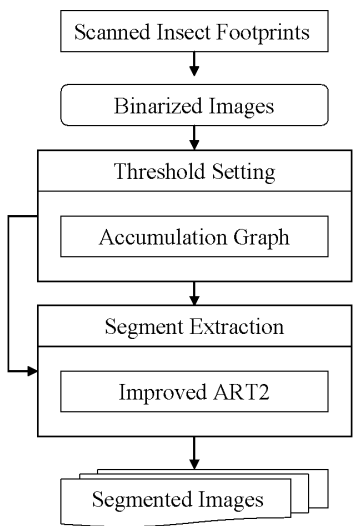

Fig. 2. Step graph of automatic insect footprint segmentation

Step 2: Collected tracking cards are scanned at 1200dpi resolution, and with 256 gray levels. The scanned images are binarized using Abutaleb's higher-order entropy algorithm, which was already identifies as being a useful method for binarization of scanned insect footprint images [6].

Step 3: An initial threshold value for ART2, a neural network algorithm for clustering, is decided using the contour shape of the graph created by accumulating distances between all the spots of a footprint pattern image.

Step 4: The decided threshold value is used in ART2, and basic segments of footprints are extracted automatically by the improved ART2 clustering algorithm regardless of size and stride of each type of insect footprint.

\subsection{Abutaleb's Higher-Order Entropy Binarization Algorithm}

Quality of the binarization process is crucial for the overall performance of recognition. Various kinds of binarization methods have been developed during the past 20 or more years; see, for example, [78. In this paper we use the binarization method proposed by Abutaleb [9] which can be briefly explained as follows:

The histogram and the probability mass function (PMF) of the image are given, respectively, by $h(g)$ and by $p(g)$, for $g=0 \ldots G$, where $\mathrm{G}$ is the maximum luminance value in the image (typically $G=255$ ). If the gray value range is not explicitly indicated as being equal to $\left[g_{\min }, g_{\max }\right]$, it is assumed to extend from 0 to $G$. The cumulative probability function is defined as follows:

$$
P(g)=\sum_{i=0}^{g} p(i)
$$

The foreground (i.e., object) and background PMFs are expressed as $P_{f}(g)$, for $0 \leq g \leq T$, and $P_{b}(g), T+1 \leq g \leq G$, respectively, where $T$ is the thresh- 
old value. The foreground and background area probabilities are calculated as follows:

$$
P_{f}(T)=P_{f}=\sum_{g=0}^{T} p(g), \quad P_{b}(T)=P_{b}=\sum_{g=T+1}^{G} p(g) .
$$

The Shannon entropy, parametrically dependent on the threshold value $T$ for the foreground and background, is formulated as:

$$
H_{f}(T)=-\sum_{g=0}^{T} p_{f}(g) \log p_{f}(g), H_{b}(T)=-\sum_{g=T+1}^{G} p_{b}(g) \log p_{b}(g)
$$

The mean and variance of the foreground and background as functions of the thresholding level $T$ are denoted as:

$$
\begin{array}{cc}
m_{f}(T)=\sum_{g=0}^{T} g \cdot p(g) & \sigma_{f}^{2}(T)=\sum_{g=0}^{T}\left[g-m_{f}(T)\right]^{2} p(g), \\
m_{b}(T)=\sum_{g=T+1}^{G} g \cdot p(g) & \sigma_{b}^{2}(T)=\sum_{g=T+1}^{G}\left[g-m_{b}(T)\right]^{2} p(g)
\end{array}
$$

Abutaleb's binarization algorithm assumes the joint entropy of two related random variables, namely, the image gray value $g$ at a pixel, and the average gray value $\bar{g}$ of a neighborhood centered at that pixel. Using the 2-D histogram $p(g, \bar{g})$, for any threshold pair $(T, \bar{T})$, one can calculate the cumulative distribution $P(T, \bar{T})$, and then define the foreground entropy as follows:

$$
H_{f}=-\sum_{i=1}^{T} \sum_{j=1}^{\bar{T}} \frac{p(g, \bar{g})}{P(T, \bar{T})} \log \frac{p(g, \bar{g})}{P(T, \bar{T})}
$$

Similarly, one can define the background region's second order entropy. Under the assumption that the off-diagonal terms, that are the two quadrants $[(0, T),(\bar{T}, G)]$ and $[(T, G),(0, \bar{T})]$ are negligible and contain elements only due to image edges and noise, the optimal pair $(T, \bar{T})$ can be found as the minimizing value of the 2-D entropy functional. In this algorithm, the following equation is used for finding an optimal threshold value:

$\left(T_{\text {opt }}, \bar{T}_{\text {opt }}\right)=\arg \min \left\{\log [P(T, \bar{T})[1-P(T, \bar{T})]]+H_{f} / P(T, \bar{T})+H_{b} /[1-P(T, \bar{T})]\right\}$ where

$$
\begin{gathered}
H_{f}=-\sum_{i=1}^{T} \sum_{j=1}^{\bar{T}} \frac{p(g, \bar{g})}{P(T, \bar{T})} \log \frac{p(g, \bar{g})}{P(T, \bar{T})} \text { and } \\
H_{b}=-\sum_{i=T+1}^{G} \sum_{j=\bar{T}+1}^{G} \frac{p(g, \bar{g})}{1-P(T, \bar{T})} \log \frac{p(g, \bar{g})}{1-P(T, \bar{T})}
\end{gathered}
$$




\subsection{Clustering Method}

Insect footprint patterns are composed of sets of segments made by insect's feet, and these segments appear in the footprint image repeatedly and dispersedly. In general, it is hard to detect segments which identify a footprint (from a scanned footprint image). Meaningful groups of regions, segments identifying a single footprint, can be extracted using specific morphological characteristics defined by species, body size, leg positions and stride of an insect (see conventional research 41510] on insects).

In this paper, we propose a method for the extraction of footprint segments using an ART2 algorithm, which is a neural network algorithm that has a good performance in clustering [112]. The ART2 algorithm can cluster footprint spots easily without any morphological features.

With the ART2 algorithm, the clustering process can be performed in real time regardless of the number of massively generated data as clusters are created dynamically. The ART2 algorithm is an unsupervised learning neural network where stability (a known weakness of conventional competitive learning algorithms) is supplemented. The ART2 algorithm automatically integrates new learning results into former learning results in order to keep former learning results. The ART2 learning algorithm used in this paper is as follows:

Step 1: The $k^{\text {th }}$ input datum is defined as $x_{k}$, and the center of the $i^{\text {th }}$ cluster is defined as $w_{i}$.

Step 2: A cluster $j^{*}$, which has a minimum distance to the new input datum $x_{k}$, is selected as the winner cluster. The distance between center of a cluster and input datum is calculated by using the Euclidean distance as shown in the following equation:

$$
\left\|x_{k}-w_{j^{*}}\right\|=\min \left\|x_{k}-w_{j}\right\|
$$

Step 3: We perform the vigilance test for an input datum. If the distance between the input datum and the winner cluster is smaller than threshold value $(\sigma)$, then this input datum is accepted as similar datum with the winner cluster, and the center of the winner cluster is updated using this input datum. If the distance between the input datum and the winner cluster is not smaller than threshold $\operatorname{value}(\sigma)$, then a new cluster is created by this input datum. This process is performed by using the following equation:

$$
\text { if }\left\|x_{k}-w_{j^{*}}\right\|<\sigma, \quad w_{j^{*}}^{\text {new }}=\frac{x_{k}+w_{j^{*}}^{\text {old }} \cdot \| \text { Cluster }_{j^{*}}^{\text {old }} \|}{\| \text { Cluster }_{j^{*}}^{\text {old }} \|+1}
$$

where $\|$ Cluster $j^{*}{ }^{*} \|$ means the number of input data included in the $j^{\text {th }}$ cluster. Step 4: Step 1 to Step 3 are repeated until no input datum remains. If the whole learning process is iterated as predefined (e.g., that the number or centers of clusters does not change anymore), the learning process is terminated. 


\subsection{Automatic Threshold Selection}

We use the ART2 algorithm, an unsupervised learning algorithm, for clustering insect footprint spots. But the threshold value $(\sigma)$ in the ART2 algorithm is set by characteristics of input data heuristically, and the threshold value is of crucial importance for the performance of clustering.

When we cluster insect footprint spots by ART2 algorithm, it is difficult to preselect an initial threshold value because the sizes of feet and strides vary with the kinds of insects. For example, the Black Cockroach, one of our test insects, has dense foot intervals, and the Native Bush Cockroach, another test insect, has sparse foot intervals. So, if we set a threshold value for the Black Cockroach to obtain good clustering results, it has an improper effect on the Native Bush Cockroach segmentation, and if we set a threshold value for the Native Bush Cockroach, it also has improper effect on the Black Cockroach processes.

In order to solve this difficulty, we used the contour shape of the graph created by accumulating distances between all the spots of a footprint pattern image for an automatic setting of a threshold value used in the ART2 algorithm. But the acquired graph (by accumulating distances) has undesirable peaks due to noisy spots; so, we applied a median filter to smooth the contour of the graph.

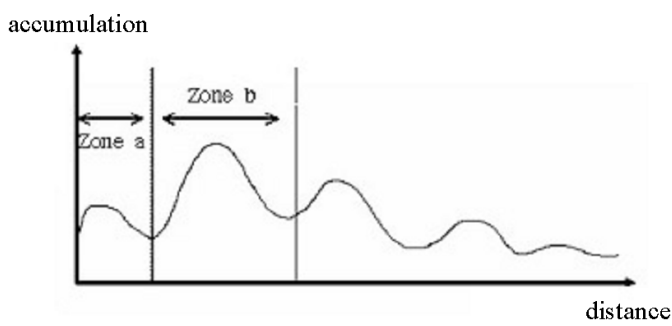

Fig. 3. Accumulation graph of distances between footprint spots

Figure 3 shows a graph of common contour shape by accumulating distances between all the spots imprinted in a general insect footprint pattern image, and

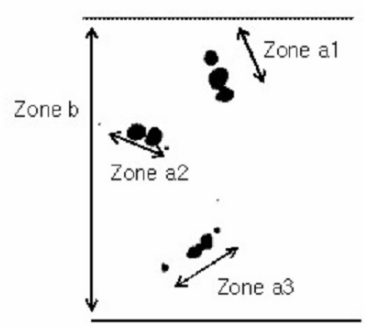

Fig. 4. A segment of an insect footprint image 
this figure represents stride and feet density of an insect. In Figure 3, "zone a" having first peak value represents accumulated distances within each front foot ("zone a1" in Figure 4), mid foot ("zone a2" in Figure 4), hind foot ("zone a3" in Figure 4), and "zone b" having second peak value represents accumulated distances between each spot in "zone b" in Figure 4. In this paper, we used the second peak value in "zone b" including front, mid, and hind legs as an initial threshold value $(\sigma)$ for accurate clustering in the ART2 algorithm.

Figure 5 shows a graph generated by a test insect footprint image, and Figure 6 shows a graph processed by a median filter in order to find the local maxima values in the graph effectively.

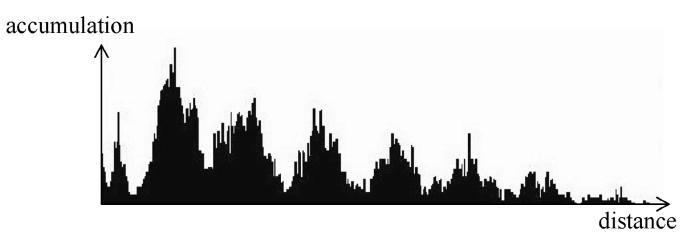

Fig. 5. Graph created by accumulating distances between all the spots

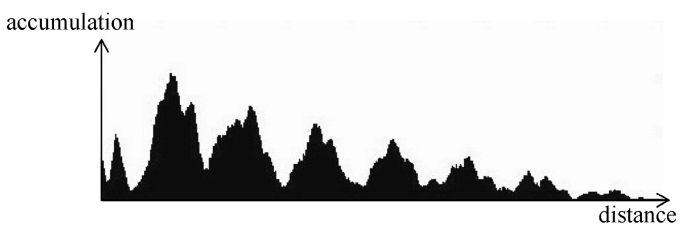

Fig. 6. Graph after applying a median filter to the graph of Figure 5

\subsection{Segment Extraction}

In this paper, we proposed a method to set an initial threshold value in the ART2 algorithm using the graph created by accumulating distances between all the spots of a footprint pattern for effective segment extraction. For segment extraction using a clustering method, the center of gravity and the size of each spot area found by linked pixels from a binarized image are utilized. The position of the center of gravity is used for center coordinates for clustering, and the size information is used for extracting final segments. Figure 7 shows a sample spot area, and also shows the center of gravity and radii (width and height) of the spot area.

$P(L, R)$ denotes the coordinates in a 2-dimensional plane of an scanned footprint image; the whole spot area is given by $P\left(L_{\min }, R_{\min }\right), P\left(L_{\max }, R_{\max }\right)$ coordinates. These coordinates are utilized for boundary coordinates in extracted segments by clustering results. Figure 8 shows each step from clustering by center of gravity and the proposed ART2 algorithm to segment extraction using size information. 


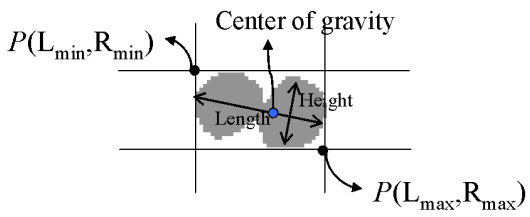

Fig. 7. Center of gravity and used radii within a spot area

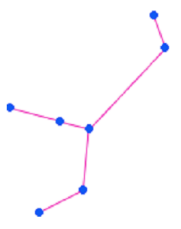

Step1

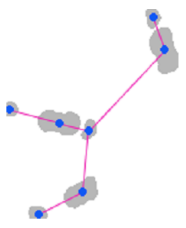

Step2

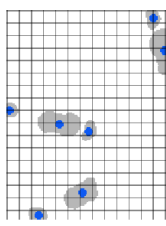

Step3

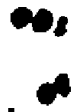

Step4

Fig. 8. Steps for segment extraction using the center of gravity and radii

\section{Experimental Results and Analysis}

We restricted experiments on two kinds of insects (Black Cockroaches and Native Bush Cockroaches) which illustrate the typical difficulty of dealing with different sizes of feet and stride lengths. Figure 9 shows 256 gray level insect footprints; the image (left) is acquired by scanning a tracking card, and the binarized image (right) is obtained by using Abutaleb's binarization algorithm. Figure 10 shows extracted segment images from a Black Cockroach footprint image using the proposed method, and Figure 11 shows extracted segment images from a Native Bush Cockroach image. Table 1 shows experimental results of segment extraction, using three scanned cards for Black Cockroaches and Native Bush Cockroaches each. If there are noisy ink traces (e.g., by the abdomen of an insect, or by insect foot dragging during tracking card acquisition), then we obtain too many noise spots in the binarized images. Some low success ratios in Table 1 are caused by such noisy spots. Thus, the next step is to develop an effective noise removal method.

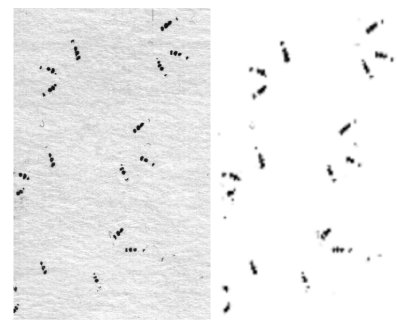

Fig. 9. A sample image (left) and the binarized image (right) using Abutaleb's algorithm 


\begin{tabular}{|c|c|c|c|}
\hline . & $\infty$ & $\therefore$ & ${ }^{\infty}$ \\
\hline 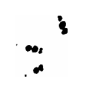 & $\infty$ &. & 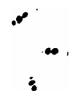 \\
\hline $\begin{array}{l}\dot{8} \\
\infty\end{array}$ & $\because$ & ! & $\cdots$ \\
\hline : & $\begin{array}{ll}\bullet & \\
\therefore & \end{array}$ & ¿ & \\
\hline
\end{tabular}

Fig. 10. Results of segment extraction for the Black Cockroach

\begin{tabular}{|c|c|c|c|}
\hline$! *$ & .. & $\stackrel{\cdot}{3}$ & $\because$ \\
\hline •. & $\ddot{*}$ & $\therefore$ & ${ }^{\circ}$ \\
\hline i. & $\therefore$ & $\because \quad$ & \\
\hline
\end{tabular}

Fig. 11. Results of segment extraction for the Native Bush Cockroach

Table 1. Results of footprint segment extraction

\begin{tabular}{ccccccc}
\hline \multirow{2}{*}{ Type of Insect } & Native Bush Cockroach & \multicolumn{3}{c}{ Black Cockroach } \\
& N1 & N2 & N3 & B1 & B2 & B3 \\
\hline Initial Threshold Value & 205 & 235 & 400 & 295 & 290 & 330 \\
Number of Test Images & 9 & 11 & 11 & 18 & 15 & 14 \\
\# of Correct Extraction & 7 & 11 & 10 & 17 & 15 & 11 \\
\# of Incorrect Extraction & 2 & 0 & 1 & 1 & 0 & 3 \\
Success Ratio & $77.8 \%$ & $100 \%$ & $90.9 \%$ & $94.4 \%$ & $100 \%$ & $78.6 \%$ \\
\hline
\end{tabular}

\section{Conclusions}

In this paper, we proposed a clustering method for extracting insect footprint segments as a preprocessing stage of insect footprint recognition. We improved the ART2 algorithm by an automatic threshold value setting (by using the 
contour shape of the graph created by accumulating distances between all the spots of footprint pattern) in the proposed clustering method. In the experiments, using two kinds of insect footprint patterns (with clearly understandable differences), the clustering results of the proposed method were almost the same as those of a human expert.

Acknowledgment. Data used in this paper were provided by the insect track recognition project at CITR, The University of Auckland. The project was initiated in 2003 by Warren Agnew (Connovation Ltd., Auckland).

\section{References}

1. Russel, J.: A recent survey of methods for closed populations of small mammals. unpublished report, The University of Auckland, Auckland (2003)

2. Whisson, D.A., Engeman, R.M., Collins, K.: Developing relative abundance techniques (RATs) for monitoring rodent population. Wildlife Research 32, 239-244 (2005)

3. Connovation Ltd.: (last visit: August 2007), see www.connovation.co.nz

4. Deng, L., Bertinshaw, D.J., Klette, R., Klette, G., Jeffries, D.: Footprint identification of weta and other insects. In: Proc. Image Vision Computing New Zealand, pp. 191-196 (2004)

5. Gray, J.: Animal Locomotion. Weidenfeld \& Nicolson, London (1968)

6. Woo, Y.W.: Performance evaluation of binarizations of scanned insect footprints. In: Klette, R., Žunić, J. (eds.) IWCIA 2004. LNCS, vol. 3322, pp. 669-678. Springer, Heidelberg (2004)

7. Rosenfeld, A., De la Torre, P.: Histogram concavity analysis as an aid in threshold selection. IEEE Transactions on System Man Cybernetics 13, 231-235 (1983)

8. Sezgin, M., Sankur, B.: Survey over image thresholding techniques and quantitative performance evaluation. J. Electronic Imaging 13, 146-165 (2004)

9. Abutaleb, A.S.: Automatic thresholding of gray-level pictures using twodimensional entropy. Computer Vision Graphics Image Processing 47, 22-32 (1989)

10. Hasler, N., Klette, R., Rosenhahn, B., Agnew, W.: Footprint recognition of rodents and insects. In: Proc. Image Vision Computing New Zealand, pp. 167-173 (2004)

11. Carpenter, G.A., Grossberg, S.: The ART of adaptive pattern recognition by a self-organizing neural network. Computer 21, 77-88 (1988)

12. Haykin, S.: Neural Networks: A Comprehensive Foundation, MacMillan (1994) 